\title{
Task Force 3 Discussions
}

\author{
Sze-leung Cheung
}

IAU Office for Astronomy Outreach, Mitaka, Tokyo, Japan

email: cheungszeleung@iau.org

\begin{abstract}
Task Force 3 is focused on public outreach. A number of projects have been funded by the $\mathrm{OAD}$ in the past, and in this meeting, the opportunity was provided for both funded projects as well as other non-funded projects with similar objectives. Three projects were presented during the session which sparked discussion in this area.The following outlines the talks.
\end{abstract}

Keywords. OAD, astronomy, development

\section{Star parties in Mexico, extended to Colombia and China}

by Silvia Torres-Peimbert, Jose Franco Institution: Universidad Nacional Autonoma de Mexico

Sparked by the enthusiasm of the International Year of Astronomy, a set of simultaneous star parties have been held since 2008 in several cities in Mexico. These star parties have raised big expectations among the population and they have been repeated at least yearly. The activity has increased in size and participating sites, which was very successful. The most recent one took place on November 29th 2014, and it included 55 locations across Mexico as well as 5 in Colombia and one in China. To organize this activity a Mexican National Committee was created formed by several universities, the French Embassy, related industries and astronomical societies.

Overall, this was a very successful initiative and triggered a great public interest in astronomy.

\section{Reflections on a Multi-stakeholder National Campaign in India around Comet ISON}

By Prajval Shastri Institution: Indian Institute of Astrophysics

Astronomy has been repeatedly demonstrated to be an effective vehicle to promote learning science by doing and to propagate a scientific temper among the public. In this spirit, the efforts undertaken in India during the international Year of Astronomy enabled the building of collaborations between professional astrophysicists, amateur astronomers, science teachers, science activists, theatre artistes and artist-designers. In the six years since, these networks have managed to not only sustain, but expand in reach, and have built focused attention of school-going children around astronomical events. The activities that were part of the build-up to both the Transit of Venus (2012) and the arrival of Comet ISON (2013) will be described.

A somewhat informal consortium led the campaign around the comet. The campaigners were enabled by open-source digitally based resource material available in both English and regional languages so that volunteers could reproduce the materials locally and to reach schools and tens of thousands of students across India. Since it involved so many schools and lack of monitoring and quality control was the major issues. 


\section{Dark Skies Africa: a Prototype Project with the IAU Office of Astronomy for Development}

by Constance Elaine Walker, Daniel Tellez, Stephen M. Pompea Institution: National Optical Astronomy Observatory

The IAU's Office of Astronomy for Development (OAD) awarded the National Optical Astronomy Observatory (NOAO) with a grant to deliver a "Dark Skies Outreach to Sub-Saharan Africa" program to institutions in 12 African countries during 2013: Algeria, Nigeria, Rwanda, Tanzania, Ghana, Zambia, South Africa, Ethiopia, Gabon, Kenya, Namibia and Senegal. The program helped students identify wasteful and inefficient lighting and provided ways to reduce consumption and to keep energy costs in check. The goal was to inspire students to be responsible stewards in helping their community safeguard one of Africa's natural resources - a dark night sky. Thirteen kits made by the NOAO Education and Public Outreach group were sent to coordinators at university, science center and planetarium-type institutions in the 12 countries and to the IAU OAD. The program's kit included complete instructional guides and supplies for six hands-on activities (e.g., on the importance of shielding lights and using energy efficient bulbs) and a project on energy conservation and responsible lighting (through energy audits). The activities were taught to the coordinators in a series of six Google+ Hangout sessions scheduled from June to mid-November. The coordinators at the institutions in turn trained local teachers in junior and senior high schools. The Google+ Hangout sessions also included instruction on carrying out evaluations. From the end of November until mid-December students from the different African countries shared final class projects (such as posters or powerpoints) on the program's website. The entire program was designed to help coordinators and educators work with students, parents and the community to identify dark sky resource, lighting and energy issues and to assess their status, efficiency and effectiveness.

Although precautions were taken to minimize difficulties with Customs, challenges arose with half of the kits, even though official invoices, letters specifying the kits were for educational purposes only and not for resale, and detailed content lists and prices were included in each kit.

Attendance by all at the Google+ Hangout sessions has also been a challenge. To accommodate schedules, 3 different dates were offered for the 1st Google+ Hangout session and 2 different dates for the subsequent Google+ Hangouts. For those unable to attend, the sessions were all recorded and placed on-line.

The third major problem was the communication and poor internet facilities across some sites.

\section{Concluding remarks}

Although these were just a few of the many projects evisaged under Task Force 3 (Astronomy for the Public) it gave the audience a taste of the type of activity in this area. The OAD continues to work closely with the IAU Office for Astronomy Outreach in order to further explore and expand on these types of projects and more. 